Int. J. Odontostomat., 6(3):337-347, 2012.

\title{
Deformidad Facial Asimétrica. Papel de la Hiperplasia Condilar
}

\author{
Asymmetric Facial Deformity. Role of Condylar Hyperplasia
}

\author{
Sergio Olate ${ }^{*, * \star} \&$ Marcio de Moraes ${ }^{* \star *}$
}

OLATE, S. \& DE MORAES, M. Deformidad facial asimétrica. Papel de la hiperplasia condilar. Int. J. Odontostomat., 6(3):337-347, 2012.

RESUMEN: Las deformidades dentofaciales (DDF) son conocidas y su diagnostico es cada vez mas frecuente; las DDF asimétricas son un grupo de enfermedades de complejo manejo debido a las condicionantes estéticas y funcionales comprometidas. La etiología es variada, aunque la hiperplasia condilar suele estar asociada en los pacientes adolescentes y adultos; su diagnostico se establece mediante análisis facial, estudio con medicina nuclear, tomografías computadorizadas y otros exámenes. La terapéutica mas aceptada hoy día consiste en la condilectomia parcial y la corrección de la deformidad facial basada en las necesidades estéticas y funcionales del paciente. El objetivo de esta revisión es determinar las condicionantes actuales asociadas al diagnostico y tratamiento de las DDF asimétricas y el papel que cumple la hiperplasia condilar en el desarrollo de estas alteraciones faciales.

PALABRAS CLAVE: asimetría facial, hiperplasia condilar, deformidad facial.

\section{INTRODUCCION}

Las deformidades dentofaciales (DDF) se caracterizan por presentarse en cerca del $5 \%$ de la población (Bailey et al., 1997) y consiste en un grupo de síntomas que determinan una deficiencia funcional y estética que limita las actividades diarias como la fonación y la masticación, llegando a generar también alteraciones en la postura cráneo cervical, dolor en la articulación temporomandibular junto a las alteraciones en las características psicológicas y las relaciones sociales del portador de la patología (Wolford \& Fields, 2000).

Dentro de las DDF mas reconocidas están las patologías sagitales caracterizadas por la deficiencia o exceso anteroposterior de maxila y/o mandíbula, patologías verticales con sus síndromes faciales corto y largo y también las patologías transversales donde destacan las compresiones maxilares o mandibulares y las asimetrías faciales (Wolford \& Fields). La asime- tría facial subclínica es común, incluso en sujetos saludable y aparentemente sin alteraciones faciales; Ferrario et al. (1994) estudiaron 40 mujeres y 40 hombres sin alteraciones faciales y determinaron asimetría facial en tejidos blandos entre el lado izquierdo y el derecho. En este tipo de pacientes, asintomáticos, Sanders et al. (2010) también observaron asimetrías en los tamaños de la rama mandibular sin identificar asimetrías en el tamaño condilar, lo cual seria explicado por la adaptación y remodelación condilar que podría mantener homogéneos los cóndilos estudiados.

La DDF asimétrica es anormal, de forma que es necesario definir su etiología, que según Pirttiniemi (1994) son consecuencia de alteraciones prenatales embriológicas (como la microsomia hemifacial), prenatales fetales (como la torticolis muscular congénita) y postnatales (como el crecimiento unilateral excesivo, infecciones, trauma).

\footnotetext{
* Profesor Asistente, División de Cirugía Oral y Maxilofacial, Facultad de Odontología, Universidad de La Frontera, Temuco, Chile.

** Investigador Posdoctoral en Cirugía Oral y Maxilofacial, División de Cirugía Oral y Maxilofacial, Facultad de Odontología de Piracicaba, Universidad Estadual de Campinas, Campinas, Brasil.

*** Profesor Asociado, División de Cirugía Oral y Maxilofacial, Facultad de Odontología de Piracicaba, Universidad Estadual de Campinas, Campinas, Brasil.
} 
El diagnóstico de estas patología es inicialmente desarrollada con el análisis facial y los estudios de imagen, que permiten identificar las asimetrías faciales luego de una evaluación de las proporciones de la cara. Desde el punto de vista del análisis facial, las DDF asimétricas (también llamadas laterognatismo o lateromentonismo) se evalúan inicialmente mediante una línea central que es trazada desde el punto glabela, pasando por el punto pronasal, hasta finalizar en el mentón, donde se identifican ambas áreas hemifaciales a fin de reconocer la diferencia en tamaño y posición que existe entre ellas (Vig \& Ellis, 1990). Del punto de vista dental, la diferencia entre líneas medias interincisivas y la oclusión invertida posterior unilateral o la mordida abierta posterior unilateral también permite definir la presencia de asimetría facial (Proffit \& Turvey, 2005). Aunque en algunas oportunidades la manifestación dental es mínima, es una deficiencia diagnostica no interpretar las características faciales que componen la base de la evaluación clínica.

Luego de reconocer la naturaleza patológica de esta enfermedad, la perpetuidad de las asimetrías faciales y su ausencia de tratamiento lleva a extensas alteraciones orofaciales de complejo escenario; consecuencias como la patología de la ATM vinculada al dolor y reabsorción condilar o las alteraciones masticatorias y de fonación son consecuencias conocidas. Además, las alteraciones psicosociales debido a la deformidad de la cara también se presentan, llevando a que exista una adaptación de la postura craneocervical a la deformidad del paciente, modificando su conducta diaria y sus condiciones generales de vida.

Estando la estética y la función comprometidas, el tratamiento de elección para las DDF asimétricas es de tipo ortodóncico-quirúrgico mediante cirugía ortognática monomaxilar o bimaxilar, asociando o no cirugías como reconstrucción de ATM, otras osteotomías faciales, instalación de prótesis de ATM y/o prótesis facial, entre otras (Kahnberg, 1997)

Un interesante estudio de Naini et al. (2012) concluyo que mientras aumenta el grado de asimetría, los sujetos determinan mayor necesidad de una cirugía que repare la estética y la función, señalando que cuando la asimetría de mentón se desvía $10 \mathrm{~mm}$ desde la línea media, existe una alta demanda por corregirlo quirúrgicamente, aunque esta demanda disminuye también de forma proporcional al aumento de la edad del paciente y a la disminución de la percepción de la estética facial. También se concluyo que con solo $5 \mathrm{~mm}$ de desvío del mentón desde la línea media, ya existe una percepción de cualquier observador de que existe una asimetría facial.

El objetivo de esta investigación es definir las características de las deformidades faciales asimétricas, sus condicionantes diagnosticas y terapéuticas y definir el rol de la hiperplasia condilar como causa postnatal para el desarrollo de las deformidades dentofaciales asimétricas.

\section{Consideraciones Morfológicas Diagnósticas de las} DDF Asimétricas. Desde una perspectiva histórica, el diagnóstico y tratamiento de las DDF tuvo un ascendente crecimiento luego de que Obwegeser en 1955 (Obwegeser, 2007) publicara sus trabajos clásicos sobre osteotomía sagital de rama mandibular, entregando estabilidad, funcionalidad y previsibilidad en el tratamiento propuesto. Las DDF asimétricas también fueron estudiadas por este mismo autor (Obwegeser \& Makek, 1986), clasificándolas en tres grupos: 1) asimetrías de componente vertical, donde no existe alteración oclusal de línea media pero se observa un crecimiento vertical de rama mandibular con mordida abierta posterior del lado afectado, 2) asimetrías de componente horizontal, donde existe alteración entre las líneas medianas interincisivas junto al desplazamiento transversal de un lado de la mandíbula hacia el lado contralateral y 3) la combinación de ambas situaciones; otras clasificaciones también han sido propuestas, aunque sin una aplicación ampliamente aceptada (Figs. 1 y 2). Hwang et al. (2007) definieron 5 grupos de pacientes con deformidad facial asimétrica en diferentes grados de compromiso; de 8 diferentes características propuestas para separar cada grupo y en tan solo 3 se logro definir claramente las diferencias de cada uno: desviación del mentón, discrepancia entre las líneas medianas apicales dentarias y diferencia vertical entre el punto gonion derecho e izquierdo. Estos puntos son vinculados claramente a la propuesta inicial de Obwegeser \& Makek en los sentidos vertical y transversal.

Las técnicas empleadas para describir las alteraciones asimétricas ha sido variada; en una de ellas, Song et al. (2007) evaluaron fotografías de pacientes y realizaron mediciones de líneas y ángulos, utilizando los planos de exocantion para determinar simetrías, demostrando su utilidad en el análisis. Por otra parte, Kim et al. (2003) examinando cráneos asimétricos determinaron que la posición y distancia entre los meato acústico externo derecho e izquierdo no era viable de ser utilizado como método de medición en asimetrías faciales asociadas a mandíbula y maxila. 


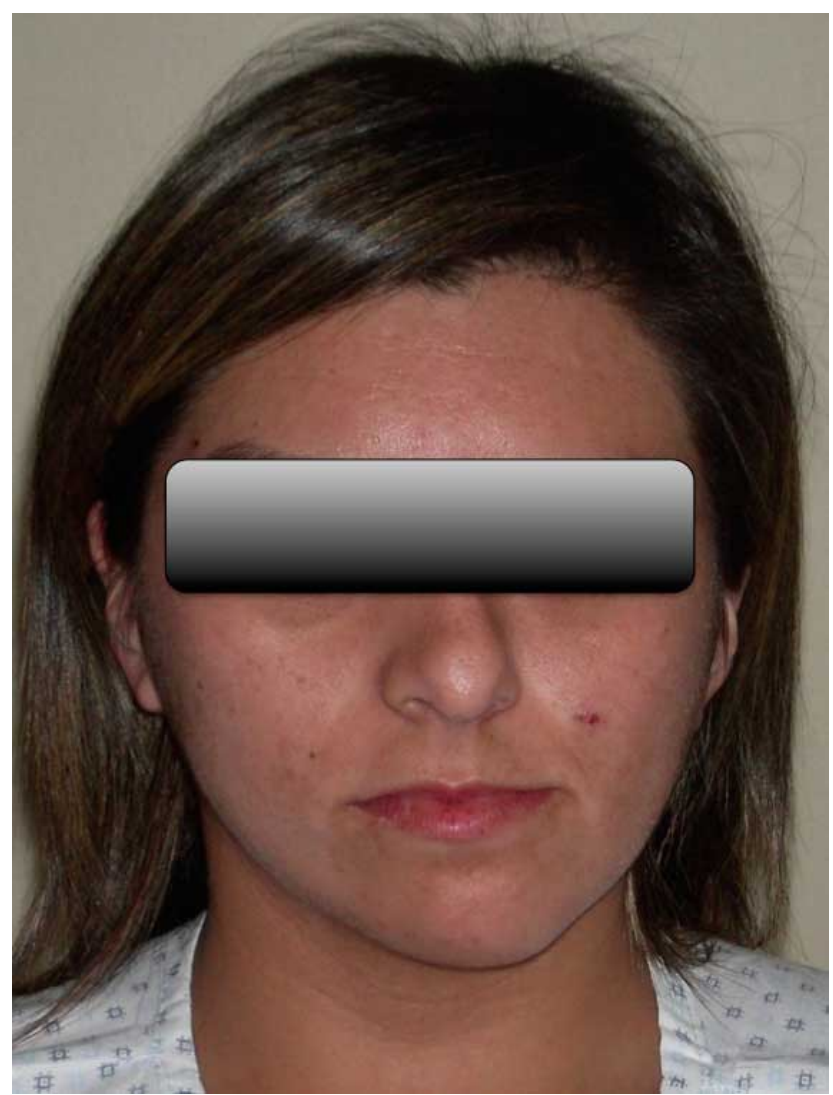

Fig. 1. Paciente con 28 años con asimetría facial y desvío de mentón de $10 \mathrm{~mm}$; es posible observar diferencias en la posición en ángulos mandibulares.

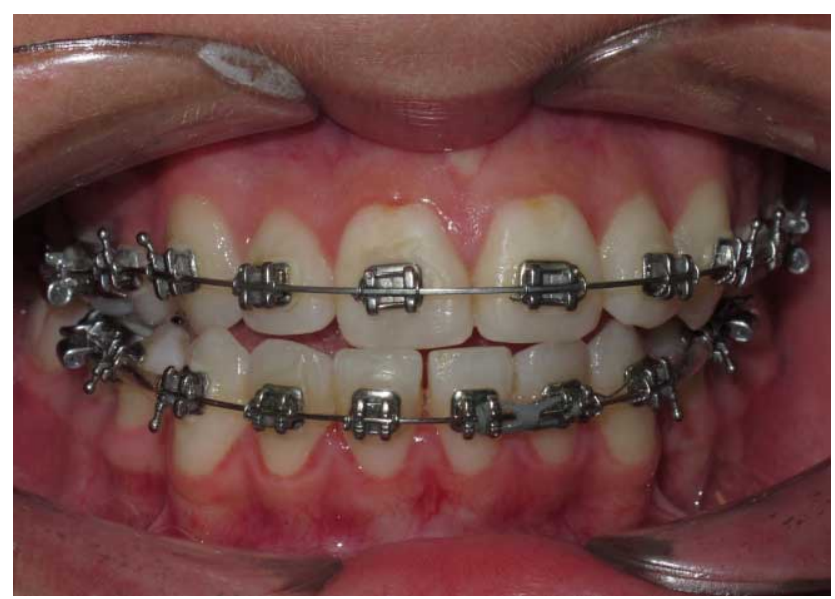

Fig. 2. Misma paciente de la Figura 1 presenta desvío de línea media dentaria inferior, compatible con la asimetría facial; paciente en preparación para realizar cirugía ortognática.

Un trabajo interesante de Maeda et al. (2006) estudio 49 pacientes (edad media 23 años) identificando que la región asimétrica mas habitual fue el cuerpo de mandíbula, seguido de la rama mandibular y la maxila, estableciendo también que el análisis con tomografía computadorizada (imagen 3D) podría remplazar los tradicionales cefalogramas con imágenes 2D. Sin embargo, Baek et al. (2007) no observaron diferencias estadísticamente significativas entre cuerpos mandibulares, pero si establecieron una inclinación mediana de un ramo mandibular pudiendo acompañarse de un crecimiento vertical excesivo de la maxila contralateral, que seria responsable de la asimetría facial.

Por otra parte, Gateno et al. (2011) comprobaron que el efecto de la asimetría en los estudios 2D o 3D depende fundamentalmente del parámetro geométrico evaluado (forma, tamaño, posición y orientación), pudiendo alterar los resultados obtenidos en el análisis $2 \mathrm{D}$ o 3D, según orientación y punto de medición. Aun así, Gateno et al. señalaron que el estudio 2D y 3D permite identificar asimetrías sutiles; de Moraes et al. (2011) mediante un estudio realizado en imágenes 2D y 3D de cráneos humanos determinaron que a pesar de que estos cráneos eran aparentemente simétricos, presentarían diferencias entre cada hemicraneo detectable en la imagen. También demostraron que en los análisis en $2 \mathrm{D}$ se pierde la relación con el análisis directo del cráneo mientras que con la imagen 3D, obtenida mediante tomografía computadorizada cone beam, se obtiene una relación mas próxima a la realidad del cráneo estudiado.

En el estudio de Sanders et al. se observaron diferencias entre las imágenes $3 D$ y $2 D$, demostrando que asimetrías faciales en el estudio 3D eran observada mientras que en el estudio 2D se observaba completa simetría entre las hemimandibulas analizadas. De esta forma, las imágenes radiográficas frontales para diagnostico no son completamente predecibles, ya que leves cambios en la posición del cráneo, en la distancia foco objeto y en el equipo pueden alterar los diagnósticos; también algunos cambios estructurales pueden generar estas alteraciones (Lee et al., 2007).

El estudio dental y oclusal también permiten definir asimetrías faciales; el canteo maxilar (desvío del plano oclusal siendo mas alto o mas bajo un lado que el otro) también es asociado a las asimetrías faciales (Hwang et al., 2009); en la detección de esta alteración es mas importante el grado del canteo que la experiencia del observador, quien no siendo un clínico entrenado, puede percibir asimetrías faciales asociadas al canteo maxilar, donde $4^{\circ}$ de canteo oclusal son detectados en el $90 \%$ de los casos, mientras que 
$3^{\circ}$ de canteo son detectados en cerca del $50 \%$ de los observadores (Padwa et al., 1997).

En este sentido, el estudio de Hwang et al. (2009) señalaron que la posición del mentón y el canteo del plano ocusal influyen significativamente en la posición del labio, mostrándolo asimétrico cuando los puntos señalados difieren de la línea media. Kwon et al. (2007) también reportan que en pacientes con asimetrías se identificaron diferencias en la arquitectura muscular en relación al músculo perigoideo medial debido a la disminución o aumento de la distancia de origen e inserción del musculo en cuestión. Músculos como el masetero, relativamente vertical, no presentaron mayor diferencia en el origen e inserción de los diferentes lados del paciente asimétrico. La angulación del músculo desde el eje axial es una de los motivos que explica esta diferencia en el músculo pterigoideo medial.

Propuestas Terapéuticas para las DDF Asimétricas. La cirugía ortognática monomaxilar o bimaxilar ha sido reconocida precozmente como una técnica segura y estable para tratar deformidades asimétricas del tercio medio e inferior de la cara (Hovinga et al., 1974). El año 1974, Brami et al. (1974) presentaron una serie de casos con osteotomías modificadas de maxila y hueso zigomático para compensar las deformidades faciales en conjunto con osteotomías mandibulares de tipo sagital de rama. Actualmente nuevas tecnologías (como la navegación intraoperatoria) son utilizadas para conseguir el mismo objetivo (Sadiq et al., 2012)

La cirugía ortognática permite modificar el canteo del plano oclusal y posicionar la línea media del mentón en su punto medio. Hwang et al. (2009) estudiaron un grupo con asimetría maxilar leve $(0,2$ $\mathrm{mm}$ ), desvío del plano oclusal y asimetría mandibular con desvío de mentón; al realizar cirugía monomaxilar (solo mandíbula) lograron recuperar el canteo oclusal, llevar el mentón a la línea mediana y mejorar significativamente la posición, corrigiendo la asimetría de tejidos blandos.

Yamashita et al. (2009) trataron 17 pacientes con asimetrías faciales donde existía una diferencia vertical de las comisuras labiales de $3,48 \mathrm{~mm}$; después de la cirugía ortognática se llego a una diferencia vertical de $0,46 \mathrm{~mm}$. La inclinación del plano labial se redujo de $2,7^{\circ}$ a $0,5^{\circ}$; esta situación se acompaño de la reposición de mentón que desde $6 \mathrm{~mm}$ de desvío llego a centrarse en tan solo $0,4 \mathrm{~mm}$ de desvío, mostrándose clínicamente simétrico.
Ko et al. (2009) señalaron que en su muestra de 27 pacientes con asimetrías faciales la gran mayoría presentaba clase III Dentofacial y fueron tratados por cirugía ortognática bimaxilar para corregir el canteo oclusal, la posición axial dentaria y la línea media del mentón. La corrección del canteo maxilar fue con leves descensos del lado que presentaba la deficiencia vertical (mediante osteotomía LeFort I), otorgando estabilidad quirúrgica en la etapa postoperatoria. Otras modificaciones fueron realizadas por Ferguson (2005), quien presento la secuencia de tres pacientes tratados por osteotomía sagital de rama extendido hasta el foramen mental en conjunto con osteotomía de la base mandibular, con lo cual se optimizo los resultados estéticos postoperatorios.

\section{DDF Asimétrica y el Papel de la Hiperplasia Condilar}

Consideraciones de la etiología. En las patologías asimétricas postnatales asociadas a patologías condilares no se observan asimetrías craneales (Baek et al.), de forma que la deformidad es generada por alteraciones posteriores a los 5 años de edad, que es el tiempo que toma la base del cráneo para tener su formación (Sgouros et al., 1993). La ATM y específicamente el cóndilo es responsable de buena parte de las asimetrías postnatales, siendo conocidas las patologías como osteocondromas, reabsorciones condilares, deficiencias en su crecimiento vinculadas a infecciones, trauma o también las denominadas hiperplasias condilares.

La hiperplasia condilar tiene una etiología desconocida y se caracteriza por presentar un crecimiento progresivo y autónomo generando un mayor volumen óseo en uncondilo que en el otro (Figs. 3 y 4). Se presenta generalmente en sujetos en fase de crecimiento, principalmente en la adolescencia (Nitzan et al., 2008). La presentación y el diagnostico de la hiperplasia condilar es un fenómeno conocido y antiguo. Norman \& Painter (1980) realizaron una revisión histórica de la patología y publicaron en el año 1980 una serie de casos que fueran descritos anteriormente por Robert Adams en el año 1836, George Humphry en el año 1856 y otros, que demuestran el conocimiento de la enfermedad y el conocimiento de la evolución de la misma, destacando la deformidad facial y el desplazamiento del mentón como una de sus principales características.

La revisión sistemática de Raijmakers et al. (2012) evaluó 10 artículos publicados con un total de 


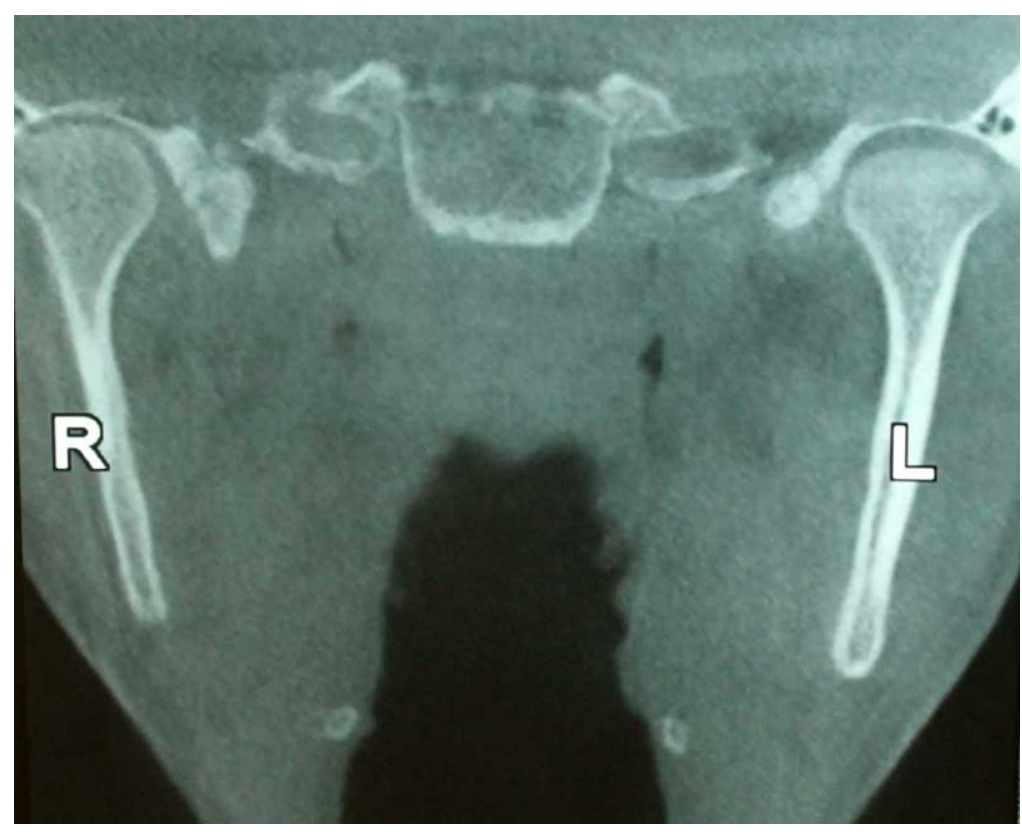

Fig. 3. Evaluación con tomografía cone beam demostrando un aumento en el tamaño del cóndilo derecho, con disminución del tamaño del área articular superior.

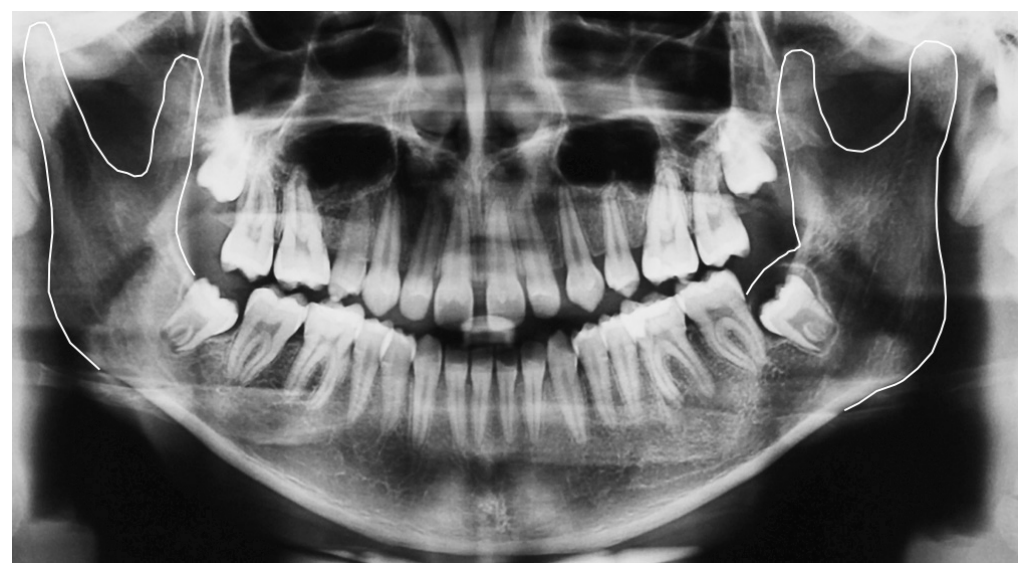

Fig. 4. Mismo paciente de la Figura 3 presentando una clara diferencia de forma y tamaño entre ambos cóndilos, ramas y ángulos mandibulares, donde el cóndilo derecho ha sido diagnosticado con hiperplasia condilar.

275 pacientes con diagnostico de hiperplasia condilar; en la muestra se observo una tendencia estadísticamente significativa a que la mujer presenta mayor hiperplasia condilar, reportando un $0,64 \%$ mas que los hombres.

Por otra parte, Nitzan et al. (2008) señalaron que la severidad de la asimetría fue estadísticamente asociado a la edad y al genero; el tipo de deformidad condilar (características morfológicas del cóndilo) no fue correlacionada con el tipo de asimetría (según Obwegeser \& Makek), presentando deformidades transversales en el $53 \%$, verticales en el $31 \%$ y combinadas en el $16 \%$. En el grupo de 36 pacientes de Villanueva-Alcojol et al. (2011) el 66,7\% presentaba patología transversal, el $22,2 \%$ vertical y el $11,1 \%$ pre- sentaba una combinación de ambos, con lo cual se observa la mayor incidencia de las patologías transversales.

\section{Cintigrama Óseo y Métodos Diagnós-}

ticos. Aunque el diagnostico de hiperplasia condilar es clínico, existen estudios de apoyo que determinan su actividad y morfología. Sin duda, la tomografía computadorizada ha contribuido a determinar la patología y la morfología condilar, permitiendo reconocer y clasificar diferentes grados de enfermedad (Mutoh et al. 1991) (Tabla I).

Dentro de los exámenes completmentarios mas utilizados para el diagnostico de la hiperplasia condilar se encuentra la cintigrafia ósea; corresponde a un examen utilizado en medicina nuclear y consiste en una exploración del esqueleto que permite detectar el metabolismo óseo. Para ello se administra Tecncio-99 junto a metilen difonato, el cual es absorbido por cristales de hidroxiapatita y calcio del tejido óseo, de forma que la intensidad de fijación es proporcional al grado de actividad osteoblástica; el examen donde se obtiene el escaneado óseo se denomina "single photon emission computed tomography" (SPECT) y determina cuantitativamente el porcentaje de absorción de un cóndilo comparándolo con el otro (Saridin et al., 2010). Diferencias de 0 a $5 \%$ de captación entre los cóndilos es observado en sujetos sin asimetrías faciales; diferencias mayores al $10 \%$ (conteo por pixel) entre ambos cóndilos ha sido considerado como crecimiento unilateral activo, siendo correlacionado positivamente con aumentos en la asimetría dental y facial del paciente (Pripatnanont et al., 2005) (Fig. 5).

Otros métodos cuantitativos no han mostrado la misma eficiencia que los resultados de la comparación entre cóndilos aportados por el SPECT (Saridin et al., 2007, 2009). Otros fluidos también han sido estudiados para determinar el crecimiento activo de un cóndilo (Laverick et al., 2009) que, utilizando la misma técni$\mathrm{ca}$, han mostrando buenos resultados. 


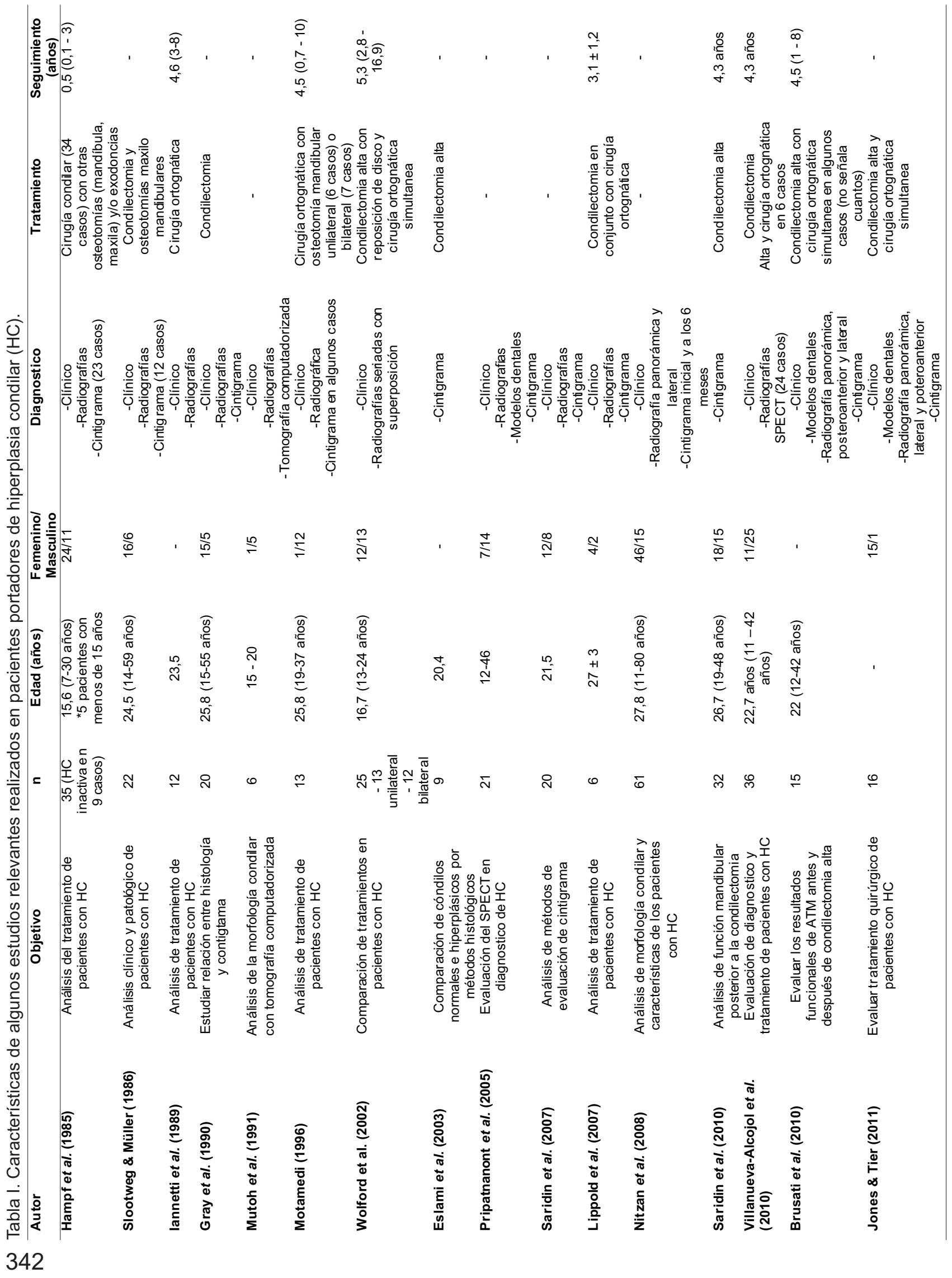




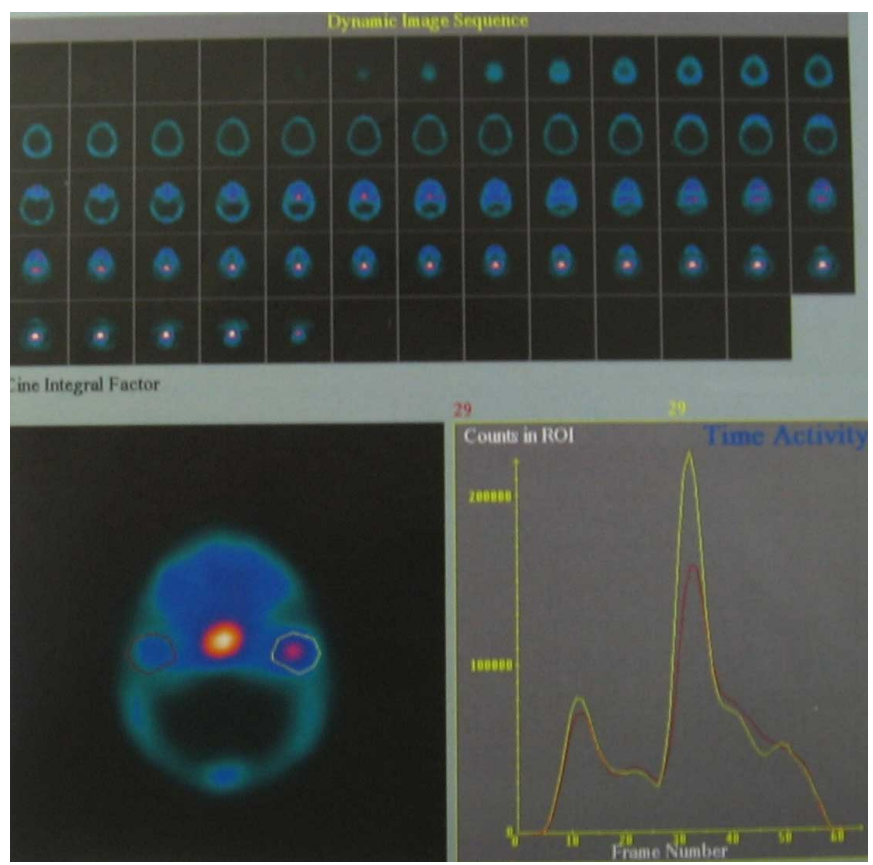

Fig. 5. Imagen de SPECT donde se observan diferentes cortes del estudio. La vista axial presenta una mayor marcación en el cóndilo derecho lo cual se asocia con mayor metabolismo óseo en la zona.

Características histológicas. Histológicamente se han caracterizado 4 capas que están presentes en la morfología condilar: 1) tejido conectivo (capa articular fibrosa), 2) capa mesenquimal indiferenciada (proliferativa), 3) capa transicional y 4) capa de cartílago hipertrófico (Hansson et al., 1977).

En 20 cóndilos de pacientes con cintigrama positivo para hiperplasia condilar, Gray et al. (1990) demostraron la presencia de una capa fibrosa de aproximadamente $0,31 \mathrm{~mm}$, luego una capa de células mesenquimales indiferenciadas con un promedio de 0,29 $\mathrm{mm}$; la capa de cartílago hipertrófico presento un promedio de $0,52 \mathrm{~mm}$. Además se observaron islas cartilaginosas al interior del tejido óseo, lo cual fue relacionado con la gravedad que presentaba el cintigrama, situación que ya había sido identificada por Slootweg \& Müller (1986), quienes también describieron una probable asociación entre el origen de la patología y el grupo etáreo del paciente, donde en la adolescencia se presentarían idiopáticamente y en la etapa adulta seria una consecuencia de tipo reactiva a alguna patología previa o trauma; lo cierto es que indistintamente de la edad del paciente, la hiperplasia condilar no tiene causa descrita y eventualmente se puede vincular a situaciones como trauma, infección u otra alteración localizada.
Otro aporte de Slootweg \& Müller fue el desarrollo de una clasificación histológica donde describieron 4 tipos de hiperplasia condilar, donde el tipo I es el mas leve y el tipo IV es el mas grave en tamaño condilar e invasión de tejidos; la clasificación se basa en la cantidad de compromiso óseo y crecimiento cartilaginoso. En este sentido, Villanueva-Alcojol et al. describieron 36 pacientes de los cuales el $44.4 \%$ presentaba tipo I, el $16,7 \%$ presentaba tipo II y el $38,9 \%$ presentaba el tipo III. Sin embargo, no fue posible obtener alguna asociación entre hallazgo histológico, hallazgo en el SPECT y la edad del paciente (Villanueva-Alcojol et al.).

Eslami et al. (2003) compararon histológicamente cóndilos sin alteraciones y cóndilos hiperplasicos, clasificados según Slootweg \& Müller, señalando que entre los cóndilos hiperplásicos se observaron diferencias significativas en la capa de cartílago hiperplasico (situada en el polo superior del cóndilo), mientras que no se observaron diferencias entre las otras capas estudiados.

El análisis realizado con AgNOR (Argyrophilic Nuclear Organizer Region) se ha dirigido a cuantificar la hiperplasia condilar, aunque por una parte Fariña et al. (2011) reportaron ausencia de correlación entre los resultados de AgNOR y la presencia de hiperplasia condilar, mientras que Eslami et al. señalo que AgNOR se asociaba a la clasificación del tipo de hiperplasia propuesta por Slootweg \& Müller, demostrando una correlación significativa.

Lippold et al. (2007), en una interesante investigación, relataron una relación directa entre los hallazgos histológicos con amplios signos de artrosis y la mayor marcación en el cintigrama, lo cual asentaría las bases de una osteotomía condilar del segmento superior del cóndilo para limitar su crecimiento anómalo.

Tratamiento de las DDF asimétricas asociadas a hiperplasia condilar. La hiperplasia condilar ha sido una reconocida causa de asimetrías faciales. Graziani (1972) presento un caso caracterizado por laterognatismo y asimetría facial que fue operado en dos tiempos quirúrgicos, siendo el primero para la resección condilar y el segundo para la resección parcial de parte del cuerpo y ángulo de mandíbula con mayor crecimiento (Tabla I).

Motamedi (1996) presentó una serie de pacientes con hiperplasia condilar diagnosticada mediante 
estudio clínico y radiográfico sin estudios cintigrama o tomografías; los pacientes fueron tratados mediantes osteotomía unilateral de mandíbula (el lado afectado), realizando una rotación mandibular a expensas del cóndilo sano. Esta técnica fue complementada en algunos casos con osteotomía LeFort I. Al no presentar un seguimiento adecuado, la principal duda esta en la estabilidad del cóndilo afectado y en el hecho de que la rotación hacia medial del cóndilo "saludable" se puede ver alterado y comprometido en su movimiento, ya que las inserciones discales y musculares presentarían un cambio en su orientación, lo que podría generar deficiencias funcionales en la ATM y una limitada estabilidad postoperatoria del movimiento ejecutado.

Otra opción terapéutica fue presentada por Choung \& Nam (1998), quienes presentaron una serie de 4 pacientes con hiperplasia condilar, donde se desarrollaba una osteotomía intraoral vertico-sagital de la rama madibular permitiendo el retiro total del segmento condilar y la remodelación del cóndilo con fresas en la mesa quirúrgica; posteriormente era nuevamente instalado en la fosa articular y estabilizado con placas de osteosíntesis; según los autores, en un seguimiento mínimo de 3 años, no se observo reabsorción acentuada del cóndilo o algún tipo de necrosis, encontrándose total funcionalidad del mismo.

El grupo de Villanueva-Alcojol et al. presentaba una edad media de 22,7 años con un rango de 11 a 42 años. En todos los pacientes fue realizada condilectomia alta (4 a $5 \mathrm{~mm}$ del polo superior del cóndilo) (Fig. 6) y en tan solo 6 de ellos fue realizada un segundo procedimiento que correspondía a cirugía ortognática para corregir el defecto facial generado. La resección de los $5 \mathrm{~mm}$ polo superior del cóndilo limita el crecimiento progresivo que presenta el cóndilo hiperplasico (Wolford et al., 2002, Lippold et al.).

La serie de pacientes de Wolford et al. (2002) demostró que la condilectomia alta en conjunto con la cirugía ortognática era previsible y aceptable para tratar el problema funcional y estético. Por otra parte, señalo que realizar la cirugía ortognática sin tratamiento condilar no limitaria el

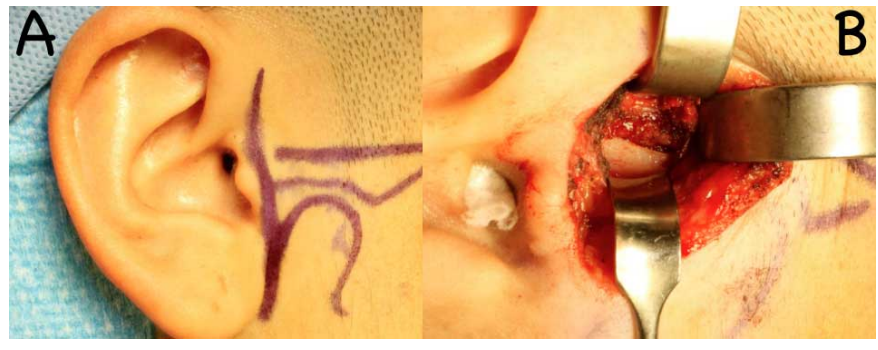

Fig. 6. Procedimiento quirúrgico clásico de condilectomia alta. A) estudio previo al acceso quirúrgico prearicular, B) condilectomia alta ejecutada sin complicaciones. crecimiento condilar posterior siendo posible la recidiva y nuevos procedimientos quirúrgicos. En este sentido Jones \& Tier (2012) presentan un estudio donde se operaron 17 pacientes tratando la patología condilar mediante condilectomia alta del lado afectado y cirugía ortognática en el mismo tiempo quirúrgico. Los autores señalan el termino "satisfactorio" para definir los resultados subjetivos postoperatorios a mediano plazo.

Uno de los pocos estudios que no preconiza la condilectomia alta como tratamiento fue realizado por lannetti et al. (1989) (que al igual de Motamedi, 1996) no presenta un adecuado diagnostico apoyado con exámenes complementarios); los autores presentaron una serie de 12 pacientes con asimetría facial asociada a hiperplasia condilar diagnosticada clínicamente y con exámenes de imagen sin uso de cintigrafia. En pacientes con una edad media de 23,5 años se realizo cirugía bimaxilar con genioplastia o osteotomía basilar de mandíbula en casi todos los casos; según los autores, la ausencia de crecimiento del cóndilo alterado justificaría no realizar la condilectomia alta; los autores también relatan seguimientos superiores a los 3 años sin recidivas, aunque en las fotografías de evaluación postoperatoria se observan asimetrías faciales que no se puede identificar si son resultado de la primera intervención o de algún crecimiento residual del cóndilo afectado.

Del punto de vista funcional, la dinámica mandibular se mantiene sin cambios significativos cuando se realiza la condilectomia alta (Wolford et al., 2002; Lippold et al.). Brusati et al. (2010) determinaron en un estudio de seguimiento de 15 pacientes sometidos a condilectomia alta que no se presentaron diferencias significativas entre la etapa preoperatoria y postoperatoria en las evaluaciones tanto objetivas como subjetivas, señalando que en el $53,3 \%$ existió una función excelente, en el $40 \%$ fue solamente buena debido a la reducción parcial de la lateralidad del lado afectado, mientras que en un paciente $(6,7 \%)$ se observó una función caracterizada como suficiente ya que este paciente abandono el tratamiento kinésico protocolar para los pacientes en la etapa postoperatoria. Saridin et al. (2010) observaron que pacientes sometidos a condilectomía por hiperplasia condilar no presentaban diferencias en desplazamiento discal y dolor miofacial al ser comparado con pacientes sin hiperplasia condilar; sin embargo, los pacientes operados presentaron mayores índices de osteoartrosis, lo cual también podría asociarse a la naturaleza de la enfermedad y no 
necesariamente a la cirugía correctiva; además, esta situación no afecto a las actividades de la vida diaria de los pacientes estudiados.

Finalmente, Wolford et al. (2002, 2009), diverge de la condicionante asimétrica de la hiperplasia condilar y destaca la existencia de una hiperplasia condilar bilateral caracterizada por el crecimiento progresivo bilateral en dirección sagital, lo cual desencadenaría una clase III Dentofacial. Si esta teoría es considerada valida, es imposible realizar una evaluación con cintigrama toda vez que este estudio evalúa comparativamente un cóndilo con el otro. Dentro de las técnicas quirúrgicas, Wolford et al. (2002, 2009) plantean la condilectomia alta bilateral en conjunto con la cirugía ortognática.

\section{CONCLUSION}

Las DDF asimétricas son conocidas y protocolizadas en su terapéutica; existe consenso para su tratamiento orto-quirúrgico donde los métodos diagnósticos de imagenbasados en cintigrama y tomografía son necesarios para el diagnóstico; aún es controversial el empleo de imagen 2D y 3D en el estudio morfológico del paciente aunque ya existen algunas orientaciones.

La hiperplasia condilar presenta controversias en su terapéutica toda vez que es difícil establecer una relación entre la morfología condilar patológica, el grado de avance de la enfermedad y su pronóstico; aunque algunos clínicos optan por esperar hasta que el crecimiento condilar del lado afectado se detiene mediante cintigramas seriados y no optan por tratar quirúrgicamente el cóndilo afectado, no es posible prever cuando ocurrirá el fin del crecimiento. Condicionados por su naturaleza desconocida, es imposible definir cuando termina el crecimiento del cóndilo hiperplasico, de forma que el crecimiento óseo mantenido de la hiperplasia condilar también permite el crecimiento muscular, ligamentoso y discal de forma asimétrica. La condilectomia alta en conjunto o no con la cirugía ortognática se visualiza como un tratamiento que previene una deformidad facial creciente sin secuelas complejas y con buenos resultados en las publicaciones estudiadas.

OLATE, S. \& DE MORAES, M. Asymmetric facial deformity. Role of condylar hyperplasia. Int. J. Odontostomat., 6(3):337-347, 2012.

ABSTRACT: Dentofacial deformities (DDF) are know and the diagnosis is ever more executed; the asymmetric DDF are a group of disease with difficult management because of esthetic a functional conditions. The etiology is these conditions is varied, although the condylar hyperplasia is frequently in adolescent and adult patients; the diagnosis is realized by facial analysis, nuclear medicine study and computed tomography and others. The more accepted therapy is related to partial condilectomy and facial deformity correction in relation to aesthetic and functional needs of patient. The aim of this review is to determine the current conditions associated to diagnosis and treatment of asymmetric DDF and the role of condylar hyperplasia in the development of these facial alterations.

KEY WORD: facial asymmetry, condylar hyperplasia, facial deformity.

\section{REFERENCIAS BIBLIOGRÁFICAS}

Baek, S. H.; Cho, I. S.; Chang, Y. I. \& Kim, M. J. Skeletodental factors affecting chin point deviation in female patients with class III malocclusion and facial asymmetry: a threedimensional analysis using computed tomography. Oral Surg. Oral Med. Oral Pathol. Radiol. Endod., 104(5):62839, 2007.

Bailey, L. J.; White, R. P. Jr.; Proffit, W. R. \& Turvey, T. A. Segmental LeFort I osteotomy for management of transverse maxillary deficiency. J. Oral Maxillofac. Surg., 55(7):728-31, 1997.

Brami, S.; Lamarche, J. P. \& Souyris, F. Treatment of facial asymmetries by one-stage maxillary and mandibular bilateral osteotomies. Int. J. Oral Surg., 3(5):239-42, 1974.
Brusati, R.; Pedrazzoli, M. \& Colletti, G. Functional results after concylectomy in active laterognathia. J. Craniomaxillofac. Surg., 38(3):179-84, 2010.

Choung, P. H. \& Nam, I. W. An intraoral approach to treatment of condylar hiperplasia or high condylar process fractures using the intraoral vertico-sagittal ramus osteotomy. J. Oral Maxillofac. Surg., 56(5):563-70; discusión 571, 1998.

de Moraes, M. E.; Hollender, L.; Chen, C. S.; Moraes, L. C. \& Balducci, I. Evaluating craniofacial asymmetry with digital cephalometric images and cone-beam computed tomography. Am. J. Orthod. Dentofacial Orthop., 139(6):e523-e531, 2011. 
Eslami, B.; Behnia, H.; Javadi, H.; Khiabani, S. \& Saffar, A. S. Histopathologic comparison of normal and hyperplastic condyles. Oral Surg. Oral Med. Oral Pathol. Oral Radiol. Endod., 96(6):711-7, 2003.

Fariña, R. A.; Becar, M.; Plaza, C.; Espinoza, I. \& Franco, M. E. Correlation between single photon emission computed tomography, AgNOR count, and histomorphologic features in patients with active mandibular condylar hyperplasia. J. Oral Maxillofac. Surg., 69(2):356-51, 2011.

Ferrario, V. F.; Sforza, C.; Poggio, C. E. \& Tartaglia, G. Distance from symmetry: A three dimensional evaluation of facial asymmetry. J. Oral Maxillofac. Surg., 52(11):1126-32, 1994.

Ferguson, J. Definitive surgical correction of the deformity resulting from hemimandibular hyperplasia. J. Craniomaxillofac. Surg., 33(3):150-7, 2005.

Gateno, J.; Xia, J. J. \& Teichgraeber, J. F. Effect of facial asymmetry on 2-Dimendional and 3-Dimensional cephalometric measurement. J. Oral Maxillofac. Surg., 69(3):655-62, 2011.

Gray, R. J.; Sloan, P.; Quayle, A. A. \& Carter, D. H. Histopathological and scintigraphic features of condylar hiperplasia. Int. J. Oral Maxillofac. Surg., 19(2):65-71, 1990.

Graziani, M. Laterognathism, supraclusion, and facial asymmetry from condylar hiperplasia. Oral Surg. Oral Med. Oral Pathol., 33(6):884-7, 1972.

Hansson, T.; Oberg, T.; Carlsson, G. E. \& Kopp, S. Thickness of the soft tissue layers and the articular disk in the temporomandibular joint. Acta Odontol. Scand., 35(2):7783, 1997.

Hampf, G.; Tasanen, A. \& Nordling, S. Surgery in mandibular condylar hiperplasia. J. Maxillofac. Surg., 13(2):74-8, 1985.

Hovinga, J.; Kraal, E. R. \& Roorda, L. A. Difficulties in and indications for the treatment of facial asymmetry. Int. J. Oral Surg., 3(5):234-8, 1974.

Hwang, H. S.; Youn, I. S.; Lee, K. H. \& Lim, H. J. Classification of facial asymmetry by cluster analysis. Am. J. Orthod. Dentofacial Orthop., 132(3):279.e1-6, 2007.

Hwang, H. S.; Min, Y. S.; Lee, S. C.; Sun, M. K. \& Lim, H. S. Change of lip-line cant after 1-jaw orthognathic surgery in patients with mandibular asymmetry. Am. J. Orthod. Dentofacial Orthop., 136(4):564-9, 2009.

lannetti, G.; Cascone, P.; Belli, E. \& Cordaro, L. Condylar hiperplasia: cephalometric study, treatment planning, and surgical correction (our experience). Oral Surg. Oral Med. Oral Pathol., 68(6):673-81, 1989.
Jones, R. H. \& Tier, G. A. Correction of facial asymmetry as a result of unilateral condylar hiperplasia. J. Oral Maxillofac. Surg., 70(6):1413-25, 2012.

Kahnberg, K. E. Correction of maxilofacial asymmetry using orthognathic surgical methods. J. Craniomaxillofac. Surg., 25(5):254-60, 1997.

Kim, Y. H.; Sato, K.; Mitani, H.; Shimizu, Y. \& Kikuchi, M. Asymmetry of the sphenoid bone and its suitability as a reference for analyzing craniofacial asymmetry. Am. J. Orthod. Dentofacial Orthop., 124(6):656-62, 2003.

Ko, E. W.; Huang, C. S. \& Chen, Y. R. Characteristics and corrective outcome of face asymmetry by orthognathic surgery. J. Oral Maxillofac. Surg., 67(10):2201-9, 2009.

Kwon, T. G.; Lee, K. H.; Park, H. S.; Ryoo, H. M.; Kim, H. J. \& Lee, S. H. Relationship between the masticatory muscles and mandibular skeleton in mandibular prognathism with and without asymmetry. J. Oral Maxillofac. Surg., 65(8):1538-43, 2007.

Laverick, S.; Bounds, G. \& Wong, W. L. [18F]-fluoride positron emission tomography for imaging condylar hyperplasia. Br. J. Oral Maxillofac. Surg., 47(3):196-9, 2009.

Lee, K. H.; Hwang, H. S.; Curry, S.; Boyd, R. L.; Norris, K. \& Baumrind, S. Effect of cephalometer misalignment on calculations of facial asymmetry. Am. J. Orthod. Dentofacial Orthop., 132(1):15-27, 2007.

Lippold, C.; Kruse-Losler, B.; Danesh, G.; Joos, U. \& Meyer, $U$. Treatment of hemimandibular hiperplasia: the biological basis of condylectomy. Br. J. Oral Maxillofac. Surg., 45(5):353-60, 2007.

Maeda, M.; Katsumata, A.; Ariji, Y.; Muramatsu, A.; Yoshida, K.; Goto, S.; Kurita, K. \& Ariji, E. 3D-CT evaluation of facial asymmetry in patients with maxilofacial deformities. Oral Surg. Oral Med. Oral Pathol. Oral Radiol. Endod., 102(3):382-90, 2006.

Motamedi, M. H. Treatment of condylar hiperplasia of the mandible using unilateral ramus osteotomies. J. Oral Maxillofac. Surg., 54(10):1161-9; discusión 1169-70, 1996.

Mutoh, Y.; Ohashi, Y.; Uchiyama, N.; Terada, K.; Hanada, K. \& Sasaki, F. Three dimensional analysis of condylar hiperplasia with computed tomography. J. Craniomaxillofac. Surg., 19(2):49-55, 1991.

Naini, F. B.; Donaldson, A. N.; McDonald, F. \& Cobourne, M. Assessing the influence of asymmetry affecting the mandible and chin point on perceived attractiveness in the orthognathic patient, clinician, and layperson. J. Oral Maxillofac. Surg., 70(1):192-206, 2012. 
Nitzan, D.; Katsnelson, A.; Bermanis, I.; Brin, I. \& Casap, N. The clinical characteristics of condylar hiperplasia: experience with 61 paients. J. Oral Maxillofac. Surg., 66(2):312-8, 2008.

Norman, J. \& Painter, D. Hyperplasia of the mandibular condyle. J. Maxillofac. Surg., 8(3):161-75, 1980.

Obwegeser, H. L. Orthognathic surgery and a tale of how three procedures came to be: a letter to the next generations of surgeons. Clin. Pastic. Surg., 34(3):331-55, 2007.

Obwegeser, H. \& Makek, M. S. Hemimandibular hiperplasia Hemimandibular elongation. J. Maxillofac. Surg., 14(4):183-208, 1986.

Padwa, B. L.; Kaiser, M. O. \& Kaban, L. B. Occlusal cant in the frontal plane as a reflection of facial asymmetry. J. Oral Maxillofac. Surg., 55(8):811-6; discussion 817, 1997.

Pirttiniemi, P. Associations of mandibular and facial asymmetries - a review. Am. J. Orthod. Dentofacial Orthop., 106(2):191-200, 1994.

Pripatnanont, P.; Vittayakittipong, P.; Markmanee, U.; Thongmak, S. \& Yipintsol, T. The use of SPECT to evaluate growth cessation of the mandible in unilateral condylar hiperplasia. Int. J. Oral Maxillofac. Surg., 34(4):364-8, 2005.

Proffit, W. \& Turvey, T. Assimetria dentofacial. En: Proffit, W.; White, R. \& Sarver, D. Tratamento Contemporâneo das Deformidades Dentofaciais. Porto Alegre: Artmed, 2005.

Raijmakers, P. G.; Karssemakers, L. H. \& Tuinzing, D. B. Female predominance and effect of gender on unilateral condylar hiperplasia: a review and meta-analysis. J. Oral Maxillofac. Surg., 70(1):e72-6, 2012.

Sadiq, Z.; Collyer, J.; Sneddon, K. \& Walsh, S. Orthognathic treatment of asymmetry: two case of "waferless" stereotactic maxillary positioning. Br. J. Oral Maxillofac. Surg., 50(2):e27-9, 2012.

Sanders, D. A.; Rigali, P. H.; Neace, W. P.; Uribe, F. \& Nanda, R. Skeletal and dental asymmetries in class II sibdivision malocclusions using cone-beam computed tomography. Am. J. Orthod. Dentofacial Orthop., 138(5):542.e1-20, 2010.

Saridin, C. P.; Raijmakers, P. \& Becking, A. G. Quantitative analysis of planar bone scintigraphy in patients with unilateral condylar hiperplasia. Oral Surg. Oral Med. Oral Pathol. Oral Radiol. Endod., 104(2):259-63, 2007.

Saridin, C. P.; Raijmakers, P. G.; Al Shamma, S.; Tuinzing, D. B. \& Becking, A. G. Comparison of different analytical methods used for analyzing SPECT scans of patients with unilateral condylar hyperactivity. Int. J. Oral Maxillofac. Surg., 38(9):942-6, 2009.
Saridin, C. P.; Gilijamse, M.; Kuik, D. L.; te Veldhuis, E. C.; Tuinzing, D.; Lobbezoo, F. \& Becking, A. G. Evaluation of temporomandibular function after high partial condilectomy because of unilateral condylar hyperactivity. J. Oral Maxillofac. Surg., 68(5):1094-9, 2010.

Slootweg, P. J. \& Müller, H. Condylar hiperplasia. A clínicopathological analysis of 22 cases. J. Maxillofac. Surg., 14(4):209-14, 1986.

Song, W. C.; Koh, K. S.; Kim, S. H.; Hu, K. S.; Kim, H. J.; Park, J. C. \& Choi, B. Y. Horizontal angular asymmetry of the face in korean Young adults with reference to the eye and mounth. J. Oral Maxillofac. Surg., 65(11):2164-8, 2007.

Sgouros, S.; Natarajan, K.; Hockely, A. D.; Goldin, J. H. \& Wake, M. Skull base growth in childhood. Pediatr. Neurosurg., 31(6):259-68, 1993.

Vig, K. D. \& Ellis, E. 3rd. Diagnosis and treatment planning for the surgical-orthodontic patient. Dent. Clin. North Am., 34(2):361-84, 1990.

Villanueva-Alcojol, L.; Monje, F. \& González-García, R. Hyperplasia of the mandibular condyle: clinical, histopathologic, and treatment considerations in a series of 36 patients. J. Oral Maxillofac. Surg., 69(2):447-55, 2011.

Wolford, L. M. \& Fields, R. T. Diagnosis and treatment planning for orthognathic surgery. In: Fonseca, R.; Betts, N. \& Turvey, T. A. (eds.). Oral and Maxillofacial Surgery: Orthognathic Surgery. Philadelphia, Saunders, 2000. pp.24-55.

Wolford, L. M.; Mehra, P.; Reiche-Fischel, O.; Morales-Ryan, C. A. \& García-Morales, P. Efficacy of high condylectomy for mangement of condylar hiperplasia. Am. J. Orthod. Dentofacial Orthop., 121(2):136-50; discussion 150-1, 2002.

Wolford LM, Morales-Ryan, C. A.; García-Morales, P. \& Perez, D. Surgical management of mandibular condylar hiperplasia type 1. Proc. (Bayl. Univ. Med. Cent.), 22(4):321-9, 2009.

Yamashita, Y.; Nakamura, Y.; Shimada, T.; Nomura, Y. \& Hirashita, A. Asymmetry of the lips of orthognathic surgery patients. Am. J. Orthod. Dentofacial Orthop., 136(4):55963, 2009.

Dirección para correspondencia:

Prof. Dr. Sergio Olate

Facultad de Odontología

Universidad de La Frontera

Claro Solar 115, Oficina 20

CHILE

Email: solate@ufro.cl

Recibido : 20-03-2012

Aceptado: 18-07-2012 